\title{
Finanzierung medizinischer Leistungen im Gefängnis
}

Schweizerische Akademie der Medizinischen Wissenschaften (SAMW)

Die Konferenzen der Kantonalen Justiz- und Polizeidirektorinnen und -direktoren (KKJPD), der Kantonalen Sozialdirektorinnen und -direktoren (SODK) sowie die Geschäftsleitung der Schweizerischen Konferenz für Sozialhilfe (SKOS) schlagen vor, dass Gesundheitskosten im Justizvollzug [1] nicht mehr als Vollzugskosten bzw. als Vollzugsnebenkosten vom Staat finanziert, sondern als persönliche Auslagen in erster Linie von der inhaftierten Person selbst getragen werden müssen [2]. Das Ostschweizer und das Nordwest- und Innerschweizer Strafvollzugskonkordat sehen vor, dass Sozialversicherungsbeiträge und durch die Krankenkasse nicht gedeckte Gesundheitskosten, soweit möglich und zumutbar, zu Lasten der eingewiesenen Person gehen [3]. Das lateinische Konkordat auferlegt der inhaftierten Person die Behandlungskosten, wenn sie aufgrund ihrer Vermögenslage oder ihres Arbeitseinkommens dazu in der Lage ist [4].

Die Zentrale Ethikkommission (ZEK) der Schweizerischen Akademie der Medizinischen Wissenschaften (SAMW) beobachtet diese Entwicklung mit Sorge. In ihren Richtlinien zur Ausübung der ärztlichen Tätigkeit bei inhaftierten Personen [5] weist die SAMW darauf hin, dass inhaftierte Personen Anrecht auf eine Gesundheitsversorgung haben, die jener der Allgemeinbevölkerung entspricht. In Bezug auf die Finanzierung dieser Leistungen sind aus Sicht der ZEK die folgenden fünf Punkte zu beachten:

1. Die Gesundheitsversorgung [6] im Freiheitsentzug ist nicht nur von zentraler Bedeutung für die inhaftierte Person selbst, sondern auch für Personen, die mit ihr Kontakt haben sowie für die Allgemeinbevölkerung.

2. Der Staat hat gegenüber inhaftierten Personen eine besondere Fürsorgepflicht. Er muss deren ausreichende Gesundheitsversorgung sicherstellen. Dabei muss er die hohe Morbidität in Anstalten und die spezifischen Gesundheitsbedürfnisse der Betroffenen berücksichtigen.

3. Inhaftierte Personen haben Anspruch auf eine Gesundheitsversorgung, die jener der Allgemeinbevölkerung gleichwertig ist. Dies gilt für alle inhaftierten Personen, unabhängig von Nationalität und Aufenthaltsstatus.
4. Inhaftierte Personen haben Anspruch auf jene Behandlung, die aus fachlicher Sicht erforderlich ist. Der medizinische Entscheid muss respektiert werden.

5. Die Gesundheitsversorgung muss für die inhaftierte Person niederschwellig zugänglich und grundsätzlich kostenfrei sein. Nur in Ausnahmefällen kann eine angemessene Kostenbeteiligung verlangt werden.

\section{Die Gesundheitsversorgung im Freiheitsent-} zug ist nicht nur von zentraler Bedeutung für die inhaftierte Person selbst, sondern auch für Personen, die mit ihr Kontakt haben und für die Bevölkerung insgesamt.

Inhaftierte Personen sind im Vergleich zur Allgemeinbevölkerung häufiger von Krankheit betroffen; oft handelt es sich um ansteckende Erkrankungen [7]. Die hohe Konzentration an gesundheitlichen Belastungen und hohe Belegungsquoten führen dazu, dass das Gefängnis per se ein belastendes Umfeld ist. Dies stellt nicht nur für die Personen im Freiheitsentzug, sondern auch für Drittpersonen ein gesundheitliches Risiko dar. Eine gute Gesundheitsversorgung schützt daher auch Mithäftlinge, Gefängnispersonal sowie Besucher und ist zudem im Interesse der Allgemeinbevölkerung, wenn die betroffene Person aus der Haft entlassen wird. Auch aus epidemiologischer Sicht drängt sich eine gute, niederschwellige Gesundheitsversorgung auf.

Bei Personen in Haft handelt es sich um eine vulnerable Patientengruppe, die im Freiheitsentzug (häufig erstmals) medizinisch erreicht werden kann. Mit einer guten Gesundheitsversorgung können Krankheiten erkannt und rechtzeitig behandelt werden. Damit kann das Risiko von Verschlechterungen des Gesundheitszustands und die Ansteckung weiterer Personen vermindert und zusätzliche Behandlungskosten vermieden werden.

2. Der Staat hat gegenüber inhaftierten Personen eine besondere Fürsorgepflicht. Er muss deren ausreichende Gesundheitsversorgung sicherstellen. Dabei gilt es, die hohe Morbidität 
in Anstalten und die spezifischen Gesundheitsbedürfnisse der Betroffenen zu berücksichtigen. Der Staat hat eine besondere Fürsorgepflicht gegenüber inhaftierten Personen, da er ihnen die Freiheit entzieht. Er trägt in diesem Sonderstatusverhältnis die Verantwortung für Leben und Gesundheit der Betroffenen und muss daher auch eine ausreichende Gesundheitsversorgung sicherstellen [8]. Studien zeigen, dass inhaftierte Personen nicht nur überdurchschnittlich von somatischen Krankheiten betroffen sind, sondern auch häufig unter psychischem Stress leiden [9].

3. Inhaftierte Personen haben Anspruch auf eine Gesundheitsversorgung, die jener der Allgemeinbevölkerung gleichwertig ist. Dies gilt für alle inhaftierten Personen, unabhängig von Nationalität und Aufenthaltsstatus.

Medizinische Leistungen in Haft müssen äquivalent sein zu denjenigen der Allgemeinbevölkerung in Freiheit [10]. Es darf keine Rolle spielen, ob die inhaftierte Person dem KVG unterstellt ist oder nicht [11]. Der Leistungskatalog gemäss KVG darf nicht unterschritten werden. Heute haben die Kantone unterschiedliche Systeme zur Kostenträgerschaft, was eine Ungleichbehandlung darstellen kann. Die ZEK empfiehlt, künftig alle inhaftierten Personen dem Krankenversicherungsobligatorium zu unterstellen.

4. Inhaftierte Personen haben Anspruch auf jene Behandlung, die aus fachlicher Sicht erforderlich ist. Der medizinische Entscheid muss respektiert werden.

Medizinische Entscheidungen, insbesondere Entscheidungen in Bezug auf die Notwendigkeit und Dringlichkeit einer medizinischen Massnahme, müssen respektiert werden. Administrative Abklärungen (z.B. vorgängige Kostengutsprache) oder sicherheitsrelevante Aspekte dürfen nicht dazu führen, dass eine medizinische Leistung, die gemäss Leistungskatalog KVG zur Verfügung steht, unterbleibt oder mit Verzögerung durchgeführt wird.

\section{Die Gesundheitsversorgung muss für die inhaftierten Personen niederschwellig zugäng- lich und grundsätzlich kostenfrei sein. Nur}

in Ausnahmefällen kann eine angemessene Kostenbeteiligung verlangt werden.

Studien zeigen, dass inhaftierte Personen teilweise zurückhaltend darin sind, medizinische Leistungen in Anspruch zu nehmen (z.B. aus Angst vor negativen Folgen einer Konsultation) [12]. Kommt als weitere Hürde hinzu, dass sie Kostenbeiträge bezahlen müssen, kann dies dazu führen, dass sie notwendige Behandlungen nicht in Anspruch nehmen.

Doch die Inanspruchnahme von medizinisch notwendigen präventiven, diagnostischen und therapeutischen Massnahmen liegt, wie dargelegt, nicht nur im Interesse der inhaftierten Personen, sondern auch im Interesse der Allgemeinheit (vgl. Punkt 1) [13]. Hürden, die dazu führen, dass Gesundheitsleistungen nicht in Anspruch genommen werden, sind daher nicht sinnvoll. Die ZEK fordert, dass die Gesundheitsversorgung für die inhaftierte Person grundsätzlich kostenfrei sein soll [14]. Nur in Ausnahmefällen, wenn ein erhebliches Einkommen und/oder hohes Vermögen vorhanden ist, soll eine angemessene Kostenbeteiligung verlangt werden können. Für die Beurteilung, ob dies zutrifft, könnten als Orientierungspunkt die SKOS-Richtlinien betreffend Verwandtenunterstützungspflicht dienen. Dass durch diese herabgesetzte finanzielle Eigenverantwortung die inhaftierte Person womöglich bessergestellt wird als die nicht inhaftierte Person, ist aufgrund der erwähnten besonderen Schutz- und Fürsorgepflichten des Staates gegenüber Menschen im Freiheitsentzug hinzunehmen.

Stellungnahme der Zentralen Ethikkommission (ZEK) der Schweizerischen Akademie der Medizinischen Wissenschaften (SAMW). Bern, 15. Februar 2019.

- Genehmigt vom Vorstand der Schweizerischen Akademie der Medizinischen Wissenschaften (SAMW). Bern, 22. Februar 2019.

- Unterstützt vom Zentralvorstand der Verbindung der Schweizer Ärztinnen und Ärzte (FMH). Bern, 13. Februar 2019.

- Unterstützt vom Vorstand der Konferenz Schweizerischer Gefängnisärzte (KSG). 28. Februar 2019.

Die Literatur zu diesem Artikel findet sich online unter www.saez.ch $\rightarrow$ Aktuelle Ausgabe oder $\rightarrow$ Archiv $\rightarrow 2019 \rightarrow 10$. 